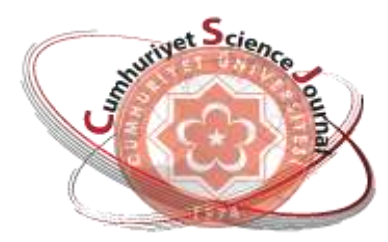

e-ISSN: 2587-246X

ISSN: 2587-2680

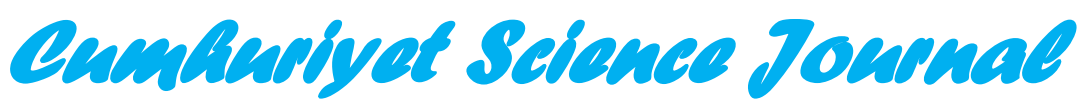

esj

Cumhuriyet Sci. J., Vol.40-1(2019) 1-10

\title{
On a Fuzzy Boundary Value Problem with an Eigenvalue Parameter Contained in the Boundary Condition
}

\author{
Hülya GüLTEKIN ÇITIL \\ Giresun University, Faculty of Arts and Sciences, Department of Mathematics, Giresun, TURKEY \\ Received: 10.01.2018; Accepted: 13.01.2019 \\ http://dx.doi.org/10.17776/csj.376952
}

\begin{abstract}
A fuzzy boundary value problem with an eigenvalue parameter contained in the boundary condition is investigated in this paper. The examination is made under the approach of Hukuhara differentiability. The effect on the eigenvalue and the eigenfunction of the problem of the eigenvalue in the boundary condition is shown.
\end{abstract}

Keywords: Fuzzy Boundary Value Problem, Hukuhara Differentiability, Eigenvalue, Eigenfunction.

\section{Sınır Koşulunda bir Özdeğer Parametre Bulunan bir Fuzzy Sınır Değer Problemi Üzerine}

Özet. Bu çalışmada sınır koşulunda bir özdeğer parametre içeren bir fuzzy sınır değer problemi araştırıldı. Bu araştırma Hukuhara diferansiyellenebilirlik yaklaşımı altında yapıldı. Sınır koşulundaki özdeğer parametrenin problemin özdeğer ve özfonksiyonu üzerindeki etkisi gösterildi.

Anahtar Kelimeler: Fuzzy Sınır Değer Problem, Hukuhara Diferansiyellenebilirlik, Özdeğer, Özfonksiyon.

\section{INTRODUCTION}

The fuzzy differential equation can be examined several approaches. The first approach is Hukuhara differentiability and for this, firstly the existence and uniqueness of the solution of a fuzzy differential equation are examined [1,2]. Gültekin and Altınış1k [3] have investigated the existence and uniqueness of solutions of two-point fuzzy boundary value problems using the Hukuhara differentiability. Gültekin Çitil and Altınışık [4] have defined the fuzzy Sturm-Liouville equation and they have examined eigenvalues and eigenfunctions of the problem under the approach of the Hukuhara differentiability.

The second approach is generalized derivative. The generalized derivative was presented in [5] and examined in [6-10]. Khastan and Nieto [11] have studied the fuzzy boundary value problem using the generalized derivative and introduced a new notion of the solution.

The third approach is to generate the fuzzy solution from the crips solution. In this approach offered by Gasilov at al. [12], a differential equation with fuzzy boundary values is investigated and the problem is interpreted as a set of crips problems.

This paper is on the eigenvalues and the eigenfunctions of the fuzzy boundary value problem with an eigenvalue parameter contained in the boundary condition under the approach Hukuhara differentiability. The drawn figures are plotted using the mathematica program. 


\section{Definition 1.1}

A function $u: \mathbb{R} \rightarrow[0,1]$ satisfying the following properties is a fuzzy number:

$\mathrm{u}$ is normal, $\mathrm{u}$ is convex fuzzy set, $\mathrm{u}$ is upper semi-continuous on $\mathbb{R}$ and $\operatorname{cl}\{x \in \mathbb{R} \mid u(x)>0\}$ is compact, where cl denotes the closure of a subset [9].

Let $\mathbb{R}_{F}$ be the space of fuzzy numbers.

\section{Definition 1.2}

Let $u \in \mathbb{R}_{F}$. The $\alpha$-level set of $u$ is $[u]^{\alpha}=\{x \in \mathbb{R} \mid u(x) \geq \alpha\}, 0<\alpha \leq 1$. If $\alpha=0$, the support of $\mathrm{u}$ is $[u]^{0}=\operatorname{cl}\{x \in \mathbb{R} \mid u(x)>0\} .[u]^{\alpha}=\left[\underline{u}_{\alpha}, \bar{u}_{\alpha}\right]$ shows the $\alpha$-level set of $u$. $\underline{u}_{\alpha}$ and $\bar{u}_{\alpha}$ denote the lower and upper branches of $[u]^{\alpha}$, respectively [11].

\section{Remark 1.1}

$\left[\underline{u}_{\alpha}, \bar{u}_{\alpha}\right]$ satisfying the following properties is the valid $\alpha$-level set:

1) $\underline{u}_{\alpha}$ is bounded monotonic increasing (nondecreasing) left-continuous function on $(0,1]$ and right-continuous for $\alpha=0$,

2) $\bar{u}_{\alpha}$ is bounded monotonic decreasing (nonincreasing) left-continuous function on $(0,1]$ and right-continuous for $\alpha=0$,

3) $\underline{u}_{\alpha} \leq \bar{u}_{\alpha}, 0 \leq \alpha \leq 1[9]$.

\section{Definition 1.3}

The $\alpha$-level set of A which is a symmetric triangular number with support $[\underline{a}, \bar{a}]$ is

$$
[A]^{\alpha}=\left[\underline{a}+\left(\frac{\bar{a}-\underline{a}}{2}\right) \alpha, \bar{a}-\left(\frac{\bar{a}-\underline{a}}{2}\right) \alpha\right][14] .
$$

\section{Definition 1.4}

The sum $u+v$ and the product $\lambda u$ are defined by $[u+v]^{\alpha}=[u]^{\alpha}+[v]^{\alpha},[\lambda u]^{\alpha}=\lambda[u]^{\alpha}, \forall \alpha \in[0,1]$, for $u, v \in \mathbb{R}_{F}$ and $\lambda \in \mathbb{R}$ [2].

The metric structure is given by the Hausdorff distance

$$
D: \mathbb{R}_{F} \times \mathbb{R}_{F} \rightarrow \mathbb{R}_{+} \cup\{0\},
$$

by

$$
\mathrm{D}(\mathrm{u}, \mathrm{v})=\sup _{\alpha \in[0,1]} \max \left\{\left|\underline{\mathrm{u}}_{\alpha}-\underline{\mathrm{v}}_{\alpha}\right|,\left|\overline{\mathrm{u}}_{\alpha}-\overline{\mathrm{v}}_{\alpha}\right|\right\}[9]
$$

\section{Definition 1.5}

Let $u, v \in \mathbb{R}_{F} . w$ which is $u=v+w, w \in \mathbb{R}_{F}$ is called the H-difference of $u$ and $v$ and it is denoted $\mathrm{u}-\mathrm{v}[14]$. 


\section{Definition 1.6}

Let $I=(a, b)$, for $a, b \in \mathbb{R}_{F}$, and $F: I \rightarrow \mathbb{R}_{F}$ be a fuzzy function. If there exist an element $F^{\prime}\left(t_{0}\right) \in \mathbb{R}_{F}$ and the limits

$$
\lim _{h \rightarrow 0^{+}} \frac{F\left(t_{0}+h\right)-F\left(t_{0}\right)}{h}=\lim _{h \rightarrow 0^{+}} \frac{F\left(t_{0}\right)-F\left(t_{0}+h\right)}{h}=F^{\prime}\left(t_{0}\right),
$$

then $\mathrm{F}$ is Hukuhara differentiable at $t_{0} \in I[9]$.

\section{Theorem 1.1}

Let $f: I \rightarrow \mathbb{R}_{F}$ be a function and show $[f(t)]^{\alpha}=\left[\underline{f}_{\alpha}(t), \bar{f}_{\alpha}(t)\right]$, for each $\alpha \in[0,1]$. If $f$ is Hukuhara differentiable, $\underline{f}_{\alpha}$ and $\bar{f}_{\alpha}$ are differentiable functions and $\left[f^{\prime}(t)\right]^{\alpha}=\left[\underline{f}_{\alpha}{ }^{\prime}(t), \bar{f}_{\alpha}{ }^{\prime}(t)\right][13]$.

\section{RESULTS AND DISCUSSION}

Consider the fuzzy Sturm-Liouville problem

$$
\begin{aligned}
& y^{\prime \prime}+\lambda y=0, x \in(a, b) \\
& \beta_{1} y(a)+\beta_{2} \lambda y^{\prime}(a)=0, \\
& \beta_{3} y(b)+\beta_{4} y^{\prime}(b)=0,
\end{aligned}
$$

where $\lambda>0, \beta_{1}, \beta_{2}, \beta_{3}, \beta_{4} \geq 0, \beta_{1}^{2}+\beta_{2}^{2} \neq 0$ and $\beta_{3}^{2}+\beta_{4}^{2} \neq 0$. Let $\lambda=k^{2}, k>0$,

$$
\begin{aligned}
\phi(x, \lambda)= & \left(k^{2} \beta_{2} \operatorname{Cos}(k a)+\frac{\beta_{1}}{k} \operatorname{Sin}(k a)\right) \operatorname{Cos}(k x)+ \\
& +\left(-\frac{\beta_{1}}{k} \operatorname{Cos}(k a)+k^{2} \beta_{2} \operatorname{Sin}(k a)\right) \operatorname{Sin}(k x)
\end{aligned}
$$

and

$$
\psi(x, \lambda)=\left(\beta_{4} \operatorname{Cos}(k b)+\frac{\beta_{3}}{k} \operatorname{Sin}(k b)\right) \operatorname{Cos}(k x)+\left(-\frac{\beta_{3}}{k} \operatorname{Cos}(k b)+\beta_{4} \operatorname{Sin}(k b)\right) \operatorname{Sin}(k x)
$$

be the solutions of the classical differential equation $y^{\prime \prime}+\lambda y=0$ satisfying the conditions $y(a)=$ $\lambda \beta_{2}, y^{\prime}(a)=-\beta_{1}$ and $y(b)=\beta_{4}, y^{\prime}(b)=-\beta_{3}$, respectively. Then,

$$
[\phi(x, \lambda)]^{\alpha}=\left[\underline{\phi}_{\alpha}(x, \lambda), \bar{\phi}_{\alpha}(x, \lambda)\right]=[\alpha, 2-\alpha] \phi(x, \lambda)
$$

and

$$
[\psi(x, \lambda)]^{\alpha}=\left[\underline{\psi}_{\alpha}(x, \lambda), \bar{\psi}_{\alpha}(x, \lambda)\right]=[\alpha, 2-\alpha] \psi(x, \lambda)
$$

are the solutions of the fuzzy differential equation (2.1) satisfying the conditions (2.2) and (2.3), respectively. The eigenvalues of the fuzzy Sturm-Liouville problem (2.1)-(2.3) if and only if are consist of the zeros of Wronskian functions

$$
\underline{W}_{\alpha}(\lambda)=W\left(\underline{\phi}_{\alpha}, \underline{\psi}_{\alpha}\right)(x, \lambda)=\underline{\phi}_{\alpha}(x, \lambda) \underline{\psi}_{\alpha}^{\prime}(x, \lambda)-\underline{\psi}_{\alpha}(x, \lambda) \underline{\phi}_{\alpha}^{\prime}(x, \lambda),
$$




$$
\bar{W}_{\alpha}(\lambda)=W\left(\bar{\phi}_{\alpha}, \bar{\psi}_{\alpha}\right)(x, \lambda)=\bar{\phi}_{\alpha}(x, \lambda) \bar{\psi}_{\alpha}^{\prime}(x, \lambda)-\bar{\psi}_{\alpha}(x, \lambda) \bar{\phi}_{\alpha}^{\prime}(x, \lambda)[4]
$$

Then,

$$
\begin{aligned}
& \underline{\phi}_{\alpha}(x, \lambda)=\alpha\left\{\left(k^{2} \beta_{2} \operatorname{Cos}(k a)+\frac{\beta_{1}}{k} \operatorname{Sin}(k a)\right) \operatorname{Cos}(k x)+\right. \\
& \left.+\left(-\frac{\beta_{1}}{k} \operatorname{Cos}(k a)+k^{2} \beta_{2} \operatorname{Sin}(k a)\right) \operatorname{Sin}(k x)\right\} \\
& \underline{\phi}_{\alpha}^{\prime}(x, \lambda)=\alpha\left\{\left(-k^{3} \beta_{2} \operatorname{Cos}(k a)-\beta_{1} \operatorname{Sin}(k a)\right) \operatorname{Sin}(k x)+\right. \\
& \left.+\left(-\beta_{1} \operatorname{Cos}(k a)+k^{3} \beta_{2} \operatorname{Sin}(k a)\right) \operatorname{Cos}(k x)\right\} \\
& \underline{\psi}_{\alpha}(x, \lambda)=\alpha\left\{\left(\beta_{4} \operatorname{Cos}(k b)+\frac{\beta_{3}}{k} \operatorname{Sin}(k b)\right) \operatorname{Cos}(k x)+\right. \\
& \left.+\left(-\frac{\beta_{3}}{k} \operatorname{Cos}(k b)+\beta_{4} \operatorname{Sin}(k b)\right) \operatorname{Sin}(k x)\right\} \\
& \psi_{\alpha}^{\prime}(x, \lambda)=\alpha\left\{\left(-\beta_{4} k \operatorname{Cos}(k b)-\beta_{3} \operatorname{Sin}(k b)\right) \operatorname{Sin}(k x)+\right. \\
& \left.+\left(-\beta_{3} \operatorname{Cos}(k b)+\beta_{4} k \operatorname{Sin}(k b)\right) \operatorname{Cos}(k x)\right\}
\end{aligned}
$$

substituing to in (2.6) and making the necessary operations yields

$$
\underline{W_{\alpha}}(\lambda)=\alpha^{2}\left(\left(\beta_{1} \beta_{4}-k^{2} \beta_{2} \beta_{3}\right) \operatorname{Cos}(k(a-b))+\left(\frac{\beta_{1} \beta_{3}}{k}+k^{3} \beta_{2} \beta_{4}\right) \operatorname{Sin}(k(b-a))\right.
$$

and

$$
\bar{W}_{\alpha}(\lambda)=(2-\alpha)^{2}\left(\left(\beta_{1} \beta_{4}-k^{2} \beta_{2} \beta_{3}\right) \operatorname{Cos}(k(a-b))+\left(\frac{\beta_{1} \beta_{3}}{k}+k^{3} \beta_{2} \beta_{4}\right) \operatorname{Sin}(k(b-a)) .\right.
$$

Then,

$$
\begin{gathered}
\underline{W}_{\alpha}(\lambda)=\bar{W}_{\alpha}(\lambda)=0 \Rightarrow\left(\left(\beta_{1} \beta_{4}-k^{2} \beta_{2} \beta_{3}\right) \operatorname{Cos}(k(a-b))+\right. \\
+\left(\frac{\beta_{1} \beta_{3}}{k}+k^{3} \beta_{2} \beta_{4}\right) \operatorname{Sin}(k(b-a))=0 .
\end{gathered}
$$

Showing $k=k_{n}$ the above equation satisfying the values $\mathrm{k}$ and substituing to in (2.4), (2.5),

$$
\begin{aligned}
{[\phi(x, \lambda)]^{\alpha}=[\alpha, 2-\alpha]\left(k_{n}{ }^{2} \beta_{2} \operatorname{Cos}\left(k_{n} a\right)+\frac{\beta_{1}}{k_{n}} \operatorname{Sin}\left(k_{n} a\right)\right) \operatorname{Cos}\left(k_{n} x\right)+} \\
+\left(-\frac{\beta_{1}}{k_{n}} \operatorname{Cos}\left(k_{n} a\right)+k_{n}{ }^{2} \beta_{2} \operatorname{Sin}\left(k_{n} a\right)\right) \operatorname{Sin}\left(k_{n} x\right) \\
{[\psi(x, \lambda)]^{\alpha}=[\alpha, 2-\alpha]\left(\beta_{4} \operatorname{Cos}\left(k_{n} b\right)+\frac{\beta_{3}}{k_{n}} \operatorname{Sin}\left(k_{n} b\right)\right) \operatorname{Cos}\left(k_{n} x\right)+}
\end{aligned}
$$




$$
+\left(-\frac{\beta_{3}}{k_{n}} \operatorname{Cos}\left(k_{n} b\right)+\beta_{4} \operatorname{Sin}\left(k_{n} b\right)\right) \operatorname{Sin}\left(k_{n} x\right)
$$

is obtained. If $[\phi(x, \lambda)]^{\alpha}$ and $[\psi(x, \lambda)]^{\alpha}$ are valid $\alpha$-level sets, they are eigenfunctions. Therefore, $[\phi(x, \lambda)]^{\alpha}$ and $[\psi(x, \lambda)]^{\alpha}$ must be valid $\alpha$-level sets. Consequently, for $k_{n}$ making $[\phi(x, \lambda)]^{\alpha}$ is a valid $\alpha$-level set, $[\phi(x, \lambda)]^{\alpha}$ is eigenfunction with associated the eigenvalues $\lambda_{n}=k_{n}^{2}$. Similarly, for $k_{n}$ making $[\psi(x, \lambda)]^{\alpha}$ is a valid $\alpha$-level set, $[\psi(x, \lambda)]^{\alpha}$ is eigenfunction with associated the eigenvalues $\lambda_{n}=k_{n}^{2}$.

\section{Example 2.1}

Consider the fuzzy boundary value problem

$$
y^{\prime \prime}+\lambda y=0, y(0)+\lambda y^{\prime}(0)=0, y(1)=0 .
$$

Let $\lambda=k^{2}, k>0$ and

$$
\begin{gathered}
\phi(x, \lambda)=k^{2} \operatorname{Cos}(k x)-\frac{1}{k} \operatorname{Sin}(k x), \\
\psi(x, \lambda)=\operatorname{Sin} k \operatorname{Cos}(k x)-\operatorname{Cos} k \operatorname{Sin}(k x)
\end{gathered}
$$

be the solutions of the classical differential equation $y^{\prime \prime}+\lambda y=0$ satisfying the conditions $y(0)=k^{2}$, $y^{\prime}(0)=1$ and $y(1)=1$, respectively. Then,

$$
\begin{gathered}
{[\phi(x, \lambda)]^{\alpha}=\left[\underline{\phi}_{\alpha}(x, \lambda), \bar{\phi}_{\alpha}(x, \lambda)\right]} \\
=[\alpha, 2-\alpha]\left(k^{2} \operatorname{Cos}(k x)-\frac{1}{k} \operatorname{Sin}(k x)\right)
\end{gathered}
$$

and

$$
\begin{gathered}
{[\psi(x, \lambda)]^{\alpha}=\left[\underline{\psi}_{\alpha}(x, \lambda), \bar{\psi}_{\alpha}(x, \lambda)\right]} \\
=[\alpha, 2-\alpha](\operatorname{Sink} \operatorname{Cos}(k x)-\operatorname{CoskSin}(k x))
\end{gathered}
$$

are the solutions of the fuzzy differential equation $y^{\prime \prime}+\lambda y=0$ satisfying the first and the second boundary conditions, respectively. Because of the eigenvalues of the fuzzy boundary value problem (2.12) are zeros of the functions $\underline{W}_{\alpha}(\lambda)$ and $\bar{W}_{\alpha}(\lambda), \underline{W}_{\alpha}(\lambda)$ and $\bar{W}_{\alpha}(\lambda)$ are obtained as

$$
\begin{gathered}
\underline{W}_{\alpha}(\lambda)=\alpha^{2}\left(-k^{2} \operatorname{Cos}(k)+\frac{1}{k} \operatorname{Sin}(k x)\right), \\
\bar{W}_{\alpha}(\lambda)=(2-\alpha)^{2}\left(-k^{2} \operatorname{Cos}(k)+\frac{1}{k} \operatorname{Sin}(k x)\right) .
\end{gathered}
$$

From this we get

$$
-k^{3} \operatorname{Cos}(k x)+\operatorname{Sin}(k x)=0 .
$$

Computing the values $\mathrm{k}$ satisfying the above equation, we have

$$
k_{1}=4.70277, k_{2}=7.85192, k_{3}=10.9948, k_{4}=14.1368, k_{5}=17.2786, \ldots
$$


Let show this values $k_{n}, n=1,2, \ldots$ and substitute in (2.13), (2.14). Then, we obtain

$$
\begin{gathered}
{\left[\phi_{n}(x)\right]^{\alpha}=\left[\underline{\phi}_{\alpha}(x),{\overline{\phi_{n}}}_{\alpha}(x)\right]=[\alpha, 2-\alpha]\left({k_{n}}^{2} \operatorname{Cos}\left(k_{n} x\right)-\frac{1}{k_{n}} \operatorname{Sin}\left(k_{n} x\right)\right)} \\
{\left[\psi_{n}(x)\right]^{\alpha}=\left[\underline{\psi}_{\alpha}(x),{\overline{\psi_{n}}}_{\alpha}(x)\right]=[\alpha, 2-\alpha]\left(\operatorname{Sin} k_{n} \operatorname{Cos}\left(k_{n} x\right)-\operatorname{Cos}_{n} \operatorname{Sin}\left(k_{n} x\right)\right)}
\end{gathered}
$$

When $\left(k_{n}{ }^{2} \operatorname{Cos}\left(k_{n} x\right)-\frac{1}{k_{n}} \operatorname{Sin}\left(k_{n} x\right)\right)>0$ and $\left(\operatorname{Sin}_{n} \operatorname{Cos}\left(k_{n} x\right)-\operatorname{Cos}_{n} \operatorname{Sin}\left(k_{n} x\right)\right)>0,\left[\phi_{n}(x)\right]^{\alpha}$ and $\left[\psi_{n}(x)\right]^{\alpha}$ are valid $\alpha$-level sets. Let be $k_{n} x \in[(n-1) \pi, n \pi], n=1,2, \ldots$.

For $\left[\phi_{n}(x)\right]^{\alpha}$,

i) If $\mathrm{n}$ is odd, $\operatorname{Sink}_{n} x \geq 0$. From here,

$$
\begin{gathered}
k_{n}{ }^{3} \operatorname{Cot}\left(k_{n} x\right) \geq 1 \Longrightarrow \operatorname{Cot}\left(k_{n} x\right) \geq \frac{1}{k_{n}{ }^{3}} \\
\Rightarrow k_{n} x \leq \cot ^{-1}\left(\frac{1}{k_{n}{ }^{3}}\right) \Longrightarrow x \leq \frac{1}{k_{n}} \cot ^{-1}\left(\frac{1}{k_{n}{ }^{3}}\right) .
\end{gathered}
$$

Since the above inequality must be for all $0 \leq x \leq 1$, it must be

$$
\frac{1}{k_{n}} \cot ^{-1}\left(\frac{1}{k_{n}^{3}}\right)-1 \geq 0 \text {. }
$$

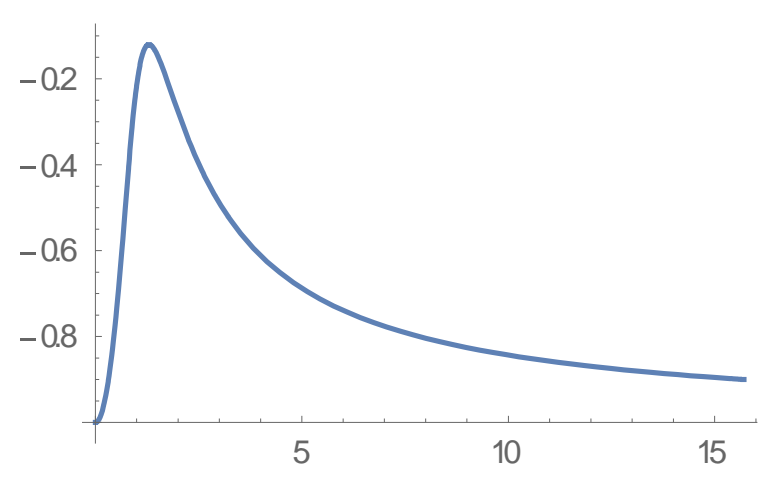

Figure 1. The graphic of the function $\frac{1}{k} \cot ^{-1}\left(\frac{1}{k^{3}}\right)-1$

ii) If $\mathrm{n}$ is even, $\operatorname{Sin}_{n} x \leq 0$. Then,

$$
\begin{gathered}
k_{n}{ }^{3} \operatorname{Cot}\left(k_{n} x\right) \leq 1 \Longrightarrow \operatorname{Cot}\left(k_{n} x\right) \leq \frac{1}{k_{n}{ }^{3}} \\
\Rightarrow k_{n} x \geq \cot ^{-1}\left(\frac{1}{k_{n}{ }^{3}}\right) \Longrightarrow x \geq \frac{1}{k_{n}} \cot ^{-1}\left(\frac{1}{k_{n}{ }^{3}}\right) .
\end{gathered}
$$

Since the above inequality must be for all $0 \leq x \leq 1$, it must be

$$
\frac{1}{k_{n}} \cot ^{-1}\left(\frac{1}{k_{n}^{3}}\right) \leq 0
$$




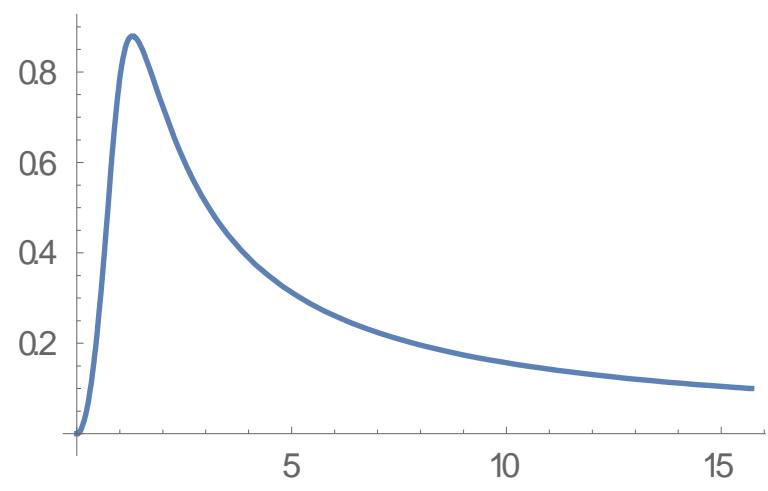

Figure 2. The graphic of the function $\frac{1}{k} \cot ^{-1}\left(\frac{1}{k^{3}}\right)$

According to Figure 1 and Figure 2, $\left[\phi_{n}(x)\right]^{\alpha}$ is not a valid $\alpha$-level set.

For $\left[\psi_{n}(x)\right]^{\alpha}$,

i) If $\mathrm{n}$ is odd, $\operatorname{Sink}_{n} x \geq 0$. Then,

$$
\begin{gathered}
\operatorname{Sin}_{n} \operatorname{Cos}\left(k_{n} x\right)-\operatorname{Cos}_{n} \operatorname{Sin}\left(k_{n} x\right) \geq 0 \\
\operatorname{Cot}\left(k_{n} x\right) \geq \operatorname{Cot}_{n} \Rightarrow k_{n} x \leq k_{n} \Rightarrow x \leq 1 .
\end{gathered}
$$

ii) If $\mathrm{n}$ is even, $\operatorname{Sin}_{n} x \leq 0$ and $\operatorname{Sink}_{n} \leq 0$. Then,

$$
\begin{gathered}
\operatorname{Sin}_{n} \operatorname{Cos}\left(k_{n} x\right)-\operatorname{Cos}_{n} \operatorname{Sin}\left(k_{n} x\right) \geq 0 \\
\operatorname{Cot}\left(k_{n} x\right) \geq \operatorname{Cot}_{n} \Rightarrow k_{n} x \leq k_{n} \Rightarrow x \leq 1 .
\end{gathered}
$$

Consequently; the eigenvalues are $\lambda_{n}=k_{n}^{2}$, with associated eigenfunctions

$$
\left[y_{n}(x)\right]^{\alpha}=[\alpha, 2-\alpha]\left(\operatorname{Sin}_{n} \operatorname{Cos}\left(k_{n} x\right)-\operatorname{Cos}_{n} \operatorname{Sin}\left(k_{n} x\right)\right) .
$$

\section{Example 2.2}

Consider the fuzzy problem

$$
y^{\prime \prime}+\lambda y=0, \quad y(0)+\lambda y^{\prime}(0)=0, y^{\prime}(1)=0
$$

Similar to example 2.1,

$$
\begin{aligned}
{[\phi(x, \lambda)]^{\alpha}=\left[\underline{\phi}_{\alpha}(x, \lambda), \bar{\phi}_{\alpha}(x, \lambda)\right] } & \\
& =[\alpha, 2-\alpha]\left(k^{2} \operatorname{Cos}(k x)-\frac{1}{k} \operatorname{Sin}(k x)\right)
\end{aligned}
$$

and

$$
\begin{aligned}
{[\psi(x, \lambda)]^{\alpha}=\left[\underline{\psi}_{\alpha}(x, \lambda)\right.} & \left., \bar{\psi}_{\alpha}(x, \lambda)\right] \\
& =[\alpha, 2-\alpha](\operatorname{Cosk} \operatorname{Cos}(k x)+\operatorname{SinkSin}(k x))
\end{aligned}
$$

are the solutions of the fuzzy differential equation $y^{\prime \prime}+\lambda y=0$ satisfying the first condition and the second condition, respectively. $\underline{W}_{\alpha}(\lambda)$ and $\bar{W}_{\alpha}(\lambda)$ are obtained as 


$$
\begin{gathered}
\underline{W_{\alpha}}(\lambda)=\alpha^{2}\left(\operatorname{Cos}(k)+k^{3} \operatorname{Sin}(k x)\right), \\
\bar{W}_{\alpha}(\lambda)=(2-\alpha)^{2}\left(\operatorname{Cos}(k)+k^{3} \operatorname{Sin}(k)\right) .
\end{gathered}
$$

From this we get

$$
\operatorname{Cos}(k x)+k^{3} \operatorname{Sin}(k x)=0 .
$$

Computing the values $\mathrm{k}$ satisfying the above equation, we have

$$
k_{1}=0.505503, k_{2}=3.10831, k_{3}=6.27915, k_{4}=9.42358, k_{5}=12.5659, \ldots
$$

Substituing this values in (2.16), (2.17), we obtain

$$
\begin{gathered}
{\left[\phi_{n}(x)\right]^{\alpha}=\left[\underline{\phi}_{\alpha}(x), \bar{\phi}_{n_{\alpha}}(x)\right]=[\alpha, 2-\alpha]\left(k_{n}{ }^{2} \operatorname{Cos}\left(k_{n} x\right)-\frac{1}{k_{n}} \operatorname{Sin}\left(k_{n} x\right)\right),} \\
{\left[\psi_{n}(x)\right]^{\alpha}=\left[{\underline{\psi_{n}}}_{\alpha}(x),{\overline{\psi_{n}}}_{\alpha}(x)\right]=[\alpha, 2-\alpha]\left(\operatorname{Cos} k_{n} \operatorname{Cos}\left(k_{n} x\right)+\operatorname{Sin} k_{n} \operatorname{Sin}\left(k_{n} x\right)\right) .}
\end{gathered}
$$

When $\left(k_{n}{ }^{2} \operatorname{Cos}\left(k_{n} x\right)-\frac{1}{k_{n}} \operatorname{Sin}\left(k_{n} x\right)\right)>0$ and $\left(\operatorname{Cos}_{n} \operatorname{Cos}\left(k_{n} x\right)+\operatorname{Sin}_{n} \operatorname{Sin}\left(k_{n} x\right)\right)>0,\left[\phi_{n}(x)\right]^{\alpha}$ and $\left[\psi_{n}(x)\right]^{\alpha}$ are valid $\alpha$-level sets. From the example 2.1, $\left[\phi_{n}(x)\right]^{\alpha}$ is not a valid $\alpha$-level set.

For $\left[\psi_{n}(x)\right]^{\alpha}$,

Let be $k_{n} x \in\left(\frac{2(n-1)-1}{2} \pi, \frac{2(n-1)+1}{2} \pi\right), n=1,3,5, \ldots$ Then, $\operatorname{Cos}\left(k_{n} x\right) \geq 0$ and

$$
\operatorname{Cos}_{n} \operatorname{Cos}\left(k_{n} x\right)+\operatorname{Sink}_{n} \operatorname{Sin}\left(k_{n} x\right) \geq 0 \Rightarrow \tan \left(k_{n}\right) \tan \left(k_{n} x\right) \geq-1 .
$$

i) If $k_{n} x \in\left(\frac{2(n-1)-1}{2} \pi,(n-1) \pi\right), n=1,3,5, \ldots, \tan \left(k_{n} x\right) \leq 0$.

$$
\begin{gathered}
\tan \left(k_{n} x\right) \leq-\frac{1}{\tan \left(k_{n}\right)} \Rightarrow k_{n} x \leq \tan ^{-1}\left(-\frac{1}{\tan \left(k_{n}\right)}\right), \\
x \leq \frac{1}{k_{n}} \tan ^{-1}\left(-\frac{1}{\tan \left(k_{n}\right)}\right) .
\end{gathered}
$$

Since the above inequality must be for all $0 \leq x \leq 1$, it must be

$$
\frac{1}{k_{n}} \tan ^{-1}\left(-\frac{1}{\tan \left(k_{n}\right)}\right)-1 \geq 0 \text {. }
$$

Figure 3. The graphic of the function $\frac{1}{k_{n}} \tan ^{-1}\left(-\frac{1}{\tan \left(k_{n}\right)}\right)-1$ 
ii) If $k_{n} x \in\left((n-1) \pi, \frac{2(n-1)+1}{2} \pi\right), n=1,3,5, \ldots, \tan \left(k_{n} x\right) \geq 0$.

$$
\begin{gathered}
\tan \left(k_{n} x\right) \geq-\frac{1}{\tan \left(k_{n}\right)} \Rightarrow k_{n} x \geq \tan ^{-1}\left(-\frac{1}{\tan \left(k_{n}\right)}\right), \\
x \geq \frac{1}{k_{n}} \tan ^{-1}\left(-\frac{1}{\tan \left(k_{n}\right)}\right) .
\end{gathered}
$$

Since the above inequality must be for all $0 \leq x \leq 1$, it must be

$$
\frac{1}{k_{n}} \tan ^{-1}\left(-\frac{1}{\tan \left(k_{n}\right)}\right) \leq 0 \text {. }
$$

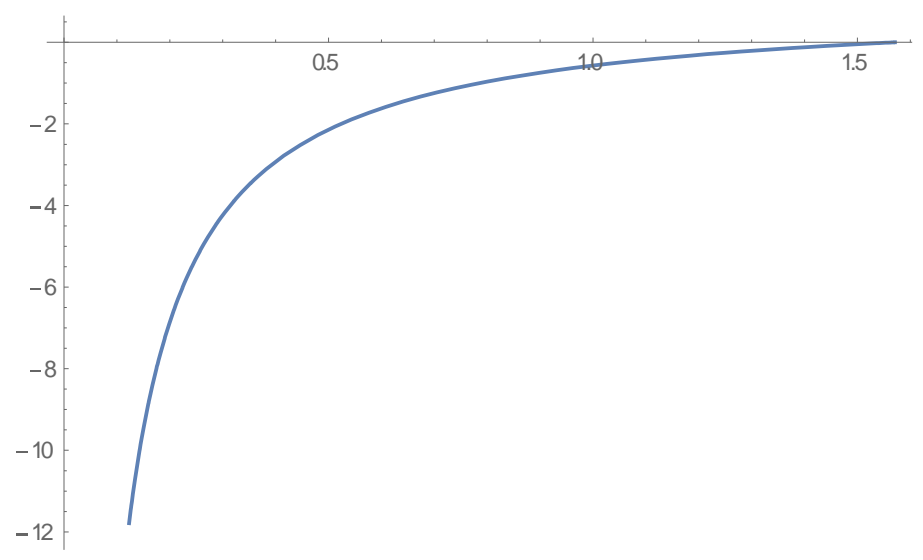

Figure 4. The graphic of the function $\frac{1}{k_{n}} \tan ^{-1}\left(-\frac{1}{\tan \left(k_{n}\right)}\right)$.

Consequently;

$k_{n} x \in\left((n-1) \pi, \frac{(2 n-1) \pi}{2}\right), \mathrm{n}=1,3,5, \ldots$. the eigenvalues are $\lambda_{n}=k_{n}^{2}$, with associated eigenfunctions

$$
\left[y_{n}(x)\right]^{\alpha}=[\alpha, 2-\alpha]\left(\operatorname{Sink}_{n} \operatorname{Cos}\left(k_{n} x\right)-\operatorname{Cos}_{n} \operatorname{Sin}\left(k_{n} x\right)\right) .
$$

\section{CONCLUSION}

A fuzzy Sturm-Liouville problem with an eigenvalue parameter contained in the boundary condition is investigated in this paper. The examination is made using the Hukuhara differentiability. The effect on the eigenvalue and the eigenfunction of the problem of the eigenvalue in the boundary condition is shown. Two examples are solved. It is shown that function that provides boundary condition with eigenvalue parameter of the differential equation does not define eigenfunction. But examples can be multiplied. Also, different studies can be made. This is a new area and these results will be useful for other mathematicians. 


\section{REFERENCES}

[1]. Buckey J.J. and Feuring T., Fuzzy Differential Equations, Fuzzy Sets and Systems, 110 (2000) 4354.

[2]. Kaleva O., Fuzzy Differential Equations, Fuzzy Sets and Systems, 24 (1987) 301-317.

[3]. Gültekin H. and Altınışık N., On Solution of Two-Point Fuzzy Boundary Value Problems, The Bulletin of Society for Mathematical Services and Standarts, 11 (2014) 31-39.

[4]. Gültekin Çitil H. and Altınışık N., On the Eigenvalues and the Eigenfunctions of the SturmLiouville Fuzzy Boundary Value Problem, Journal of Mathematical and Computational Science, 7(4) (2017) 786-805.

[5]. Bede B. and Gal S.G., Almost Periodic Fuzzy-Number-Valued Functions, Fuzzy Sets and Systems, 147 (2004) 385- 403.

[6]. Bede B. and Gal S.G., Generalizations of the Differentibility of Fuzzy Number Value Functions with Applications to Fuzzy Differential Equations, Fuzzy Sets and Systems, 151 (2005) 581-599.

[7]. Bede B., Rudas I.J and Bencsik A.L., First Order Linear fuzzy Differential Equations Under Generalized Differentiability, Inform. Sci., 177 (2007) 1648-1662.

[8]. Chalco-Cano Y. and Roman-Flores H., On New Solutions of Fuzzy Differential Equations, Chaos, Solitons \& Fractals, 38 (2008) 112-119.

[9]. Khastan A., Bahrami F. and Ivaz K., New Results on Multiple Solutions for Nth-Order Fuzzy Differential Equations under Generalized Differentiability, Boundary Value Problems, (2009) doi:10.1155/2009/395714. Article ID 395714, 13 pages.

[10]. Nieto J.J., Khastan A. and Ivaz K., Numerical Solution of Fuzzy Differential Equations under Generalized Differentiability, Nonlinear Analysis: Hybrid Systems, 3 (2009) 700-707.

[11]. Khastan A. and Nieto J.J., A Boundary Value Problem for Second Order Fuzzy Differential Equations, Nonlinear Analysis, 72 (2010) 3583-3593.

[12]. Gasilov N.A., Amrahov Ş.E. and Fatullayev A.G., A Geometric Approach to Solve Fuzzy Linear Systems of Differential Equations, Appl. Math. Inf. Sci., 5 (2011) 484-495.

[13].Fard O.S., Esfahani A. and Kamyad A.V., On Solution of a Class of Fuzzy BVPs, Iranian of Fuzzy Systems, 9-1 (2012) 49-60.

[14].Liu H.K., Comparation Results of Two-Point Fuzzy Boundary Value Problems, International Journal of Computational and Mathematical Sciences, 5-1 (2011) 1-7. 